\title{
Patients With Type 2 Diabetes at Risk for Major Depressive Disorder Over Time
}

Diana M. Naranjo, PbD

Lawrence Fisher, PbD

Patricia A. Areán, PbD

Danielle Hessler, PbD

Joseph Mullan, $\mathrm{PbD}$

Department of Family \& Community Medicine, University of California, San Francisco, San Francisco, California
Conflicts of interest: authors report none.

\section{CORRESPONDING AUTHOR}

Diana Naranjo, PhD

Department of Family \&

Community Medicine

University of California,

San Francisco, Box 0900

San Francisco, CA 94143

diana.naranjo@ucsf.edu

\begin{abstract}
PURPOSE We wanted to identify risk factors associated with the development of major depressive disorder (MDD) among patients with type 2 diabetes over time.

METHODS In a noninterventional study, 338 adult patients with type 2 diabetes and no MDD diagnosis at baseline were assessed 3 times during 18 months (9-month intervals) to ascertain predictors of MDD. We tested a model incorporating personal, behavioral, biologic, and psychosocial variables to identify predictors of MDD. Exploratory analyses tested whether current negative affect mediated the relationship between predictors and subsequent MDD. We also conducted a stratified analysis of moderate vs high negative affect to explore whether level of baseline negative affect mediated the relationship between specific predictors and MDD.

RESULTS Prior MDD and negative affect predicted future development of MDD. In subpopulations stratified by moderate negative affect, negative life events, an elevated body mass index (BMI), prior MDD, and poor control of glycated hemoglobin (hemoglobin $A_{l c}$ ) each predicted MDD. In subpopulations stratified by elevated negative affect, negative life events and poor control of hemoglobin $A_{1 c}$ predicted MDD. Current negative affect partially mediated the relationship between prior MDD and subsequent MDD, as well as the relationship between negative life events and subsequent MDD.
\end{abstract}

CONCLUSIONS Although negative affect at baseline was the primary predictor of subsequent MDD, when stratified by negative affect, negative life events, BMI, and poor control of hemoglobin $A_{1 c}$ also predicted MDD. Thus, life stresses and patients' disease-related concerns are important when understanding what predicts subsequent MDD. Addressing depressive symptoms and broader life context issues expands the scope of a potential intervention to reduce the risk of developing MDD in persons with type 2 diabetes.

Ann Fam Med 2011;9:115-120. doi:10.1370/afm.1212.

\section{INTRODUCTION}

$\mathrm{D}$ epression is a common comorbidity among patients with type 2 diabetes. It is associated with increased health care costs, disability, functional impairment, and mortality., ${ }^{1,2}$ Patients with type 2 diabetes are $52 \%$ more likely to develop major depressive disorder (MDD) than the general population, ${ }^{3}$ and most of these patients are managed in primary care. ${ }^{4}$

Although several studies have suggested all patients with diabetes be screened for MDD, 5,6 the ability to identify subsets of patients with type 2 diabetes who are at risk would channel scarce resources and focus attention on a vulnerable population. ${ }^{7,8}$ We undertook this study in an effort to identify biologic, behavioral, and psychosocial characteristics at baseline that predict the onset of MDD among primary care patients with diabetes.

\section{METHODS}

Our study was of a 3 -wave, 18 -month, noninterventional, longitudinal design that included a primary care sample of 506 patients with type 2 
diabetes. We first selected patients with no MDD at the start of the study who subsequently met Diagnostic and Statistical Manual of Mental Diseases (4th Edition) (DSM$I V)$ criteria for MDD at either 9 or 18 months later.

We recruited patients with type 2 diabetes from 4 community-based medical groups and 4 diabetes education centers in the San Francisco Bay Area. Inclusion criteria comprised the following: patients with type 2 diabetes for at least 12 months, aged 21 to 75 years, read and speak English or Spanish fluently, no severe diabetes complications (eg, retinopathy and nephropathy), and no diagnosis of psychosis or dementia. Potential participants were sent letters informing them of the study and that a project representative would call them to determine whether they were eligible and to provide more information, unless the participant opted out with a returned postcard or a telephone call to an 800 telephone number. If the patient was eligible and interested, a live visit at the patient's home, the project office, or a convenient community setting was scheduled. At that visit, more information was provided, informed consent signed, and baseline assessment completed. Participants received 2 more assessments at 9-month intervals during the next 18 months. At all 3 assessments, participants received a 1.5 -hour visit that included a brief interview, physical measurements, and a 150 -item mail-back questionnaire; they then visited a community laboratory for collection of blood and urine specimens. All materials were prepared in English and Spanish, and research assistants were fluent in both languages. The project was approved by the institutional review boards at the University of California, San Francisco, and at each participating facility. A more detailed description of recruitment methods and procedures has been reported..$^{9}$

\section{Measures}

The 4 blocks of variables identified as potential predictors of developing MDD over time were assessed at each of the 3 time points. Block 1 (demographics) included patient age, sex, income, and self-identified race (white/nonwhite). Block 2 (behavioral variables) included the diet and exercise components of the Summary of Diabetes Self-Care Activities. ${ }^{10}$ Items elicited information about diabetes self-care activities during the past 7 days pertaining to dietary recommendations (eg, "On how many of the last 7 days have you followed a healthful eating plan?"), as well as exercise recommendations (eg, "On how many of the last 7 days did you participate in at least 30 minutes of physical activity?"). Block 3 (biologic variables) included glycated hemoglobin (hemoglobin $\mathrm{A}_{1 \mathrm{c}}$ ) level, body mass index (BMI), number of comorbidities (from a list of 25), and diabetes complications. Block 4 (psychosocial variables) included several emotion-focused measures. Indicators of negative affect during the last week were assessed by the Center for Epidemiological Studies-Depression Scale (CES-D), ${ }^{11}$ a 20 -item scale $(\alpha=.89)$, with a score of 10 or greater indicating moderately elevated negative affect and a score of 16 or greater as highly elevated negative affect. Life stress was assessed by the Negative Life Events Scale (NLE), ${ }^{12}$ based on a list of 22 potential stressful events, such as the death of a friend or being a crime victim. Current and prior MDD were measured by the Composite International Diagnostic Interview (CIDI). ${ }^{13}$ A CIDI-certified trainer instructed research assistants, who attended weekly supervisory sessions, and who scored standard protocols throughout the course of the study to prevent rater drift over time. We report prior MDD as any previous depressive episode across the lifetime assessed at baseline. We report past year prevalence of MDD for baseline and "since we saw you last" at 9 months and 18 months.

We tested whether prediction was better when separating those who developed MDD at 9 or 18 months (baseline predicting MDD at 9 or 18 months), baseline predicting MDD at 9 months alone, baseline predicting MDD at 18 months alone, and MDD at 9 months predicting MDD at 18 months alone. There were no differences in the pattern of results across the 4 models tested. We therefore report findings for all patients regardless of when MDD occurred at 9 and 18 months.

\section{Data Analyses}

Applying the strategy detailed by Hosmer and Lemeshow ${ }^{14}$ we used logistic regression for all analyses with SPSS 18 (Statistical Package for the Social Sciences, SPSS Inc, Chicago, Illinois). Because of the number of variables assessed, we used hierarchical progression across models to trim the final model to a small number of significant baseline variables that predicted MDD onset at 9 or 18 months after study entry. We ran an analysis of each of the 4 blocks separately. Within each, we retained significant variables at $P<.10$ and then reassessed each model, this time including only the reduced number of significant variables, using a backward selection method. We created a combined model that included the best predictors from the final analysis of each of the 4 previous models, with patient demographics included. At each stage we assessed for nonlinear effects among continuous variables, multicollinearity, unusual changes in coefficients, and large standard errors.

We additionally explored whether different predictors of MDD emerged for patients with moderate or high levels of negative affect at baseline. We repeated the final model stratifying by 2 subgroups of patients: those with baseline CES-D scores of 10 or greater and 
those with baseline CES-D scores of 16 or greater. We tested whether any of the predictors in the combined model interacted with CES-D to predict MDD differentially. Finally, we tested whether the level of current negative affect mediated the relationship between the study predictors and the development of MDD using the analytic strategy laid out by Baron and Kenny. ${ }^{15}$ The results are presented as odds ratios.

\section{RESULTS}

Screening identified 640 eligible patients, and 506 patients completed the baseline assessment, for a

\section{Table 1. Characteristics of the Study Cohort $(\mathrm{N}=338)$}

\begin{tabular}{|c|c|}
\hline Characteristic & All Cases \\
\hline Age, mean (SD), y & $58 \pm 9.88$ \\
\hline Female, n (\%) & $254(56.4)$ \\
\hline White race, $\mathrm{n}(\%)$ & $158(35.1)$ \\
\hline Income, mean (SD), \$ (thousands) & $52.7 \pm 36.0$ \\
\hline Diet adherence last week, mean (SD), d & $4.2 \pm 1.54$ \\
\hline Exercise adherence last week, mean (SD), $d$ & $3.2 \pm 2.30$ \\
\hline Hemoglobin $A_{1 c}$ mean (SD), \% & $7.3 \pm 1.46$ \\
\hline Body mass index, mean (SD), kg/m² & $32.5 \pm 7.62$ \\
\hline Comorbidities, mean (SD), $\mathrm{n}$ & $3.7 \pm 2.42$ \\
\hline Diabetes complications, a mean (SD), $n$ & $0.8 \pm 1.22$ \\
\hline Prior major depressive disorder, $\mathrm{n}(\%)$ & $94(20.9)$ \\
\hline Negative life events in past 12 mo, mean (SD), $n$ & $3.5 \pm 3.05$ \\
\hline Negative affect at baseline, mean (SD), $\mathrm{n}$ & $9.9 \pm 9.97$ \\
\hline
\end{tabular}

$79.0 \%$ acceptance rate of eligible screened patients. Of these patients, 411 completed all 3 assessments (the mean between-assessment interval was 9.1 months). Three hundred thirty-eight patients did not meet criteria for current MDD at baseline, forming the sample for the current analyses (Table 1). Of these 338 patients, 44 patients (12\%) had MDD diagnosed at 9 or 18 months after baseline. Specifically, 21 patients had MDD diagnosed at 9 months, 16 patients at 18 months, and 7 patients at both 9 and 18 months. Those with MDD, high negative affect, or more negative life events did not miss a 9- or 18 -month assessment or drop out more often than those without these conditions. A more detailed description of attrition analyses has been reported previously. ${ }^{16}$

\section{Risk Factors for MDD \\ Block Analyses}

Results from the logistic regression of the patient demographic block (Table 2) showed that being younger $(\mathrm{OR}=0.95 ; \mathrm{P}<.05)$ and having lower incomes $(\mathrm{OR}=0.99 ; P<.05)$ significantly and independently predicted MDD at a subsequent study assessment; however, patient sex and race did not. Results from the behavioral block showed that neither diet nor exercise level significantly predicted subsequent MDD. Results from the biologic block showed that higher levels of hemoglobin $\mathrm{A}_{1 \mathrm{c}}(\mathrm{OR}=1.22 ; \mathrm{P}<.05)$ and $\mathrm{BMI}$ $(\mathrm{OR}=1.04 ; P<.05)$ significantly and independently predicted MDD over time, whereas the number of comorbidities and complications did not. Results from the psychosocial block showed that having had MDD

\section{Table 2. Adjusted Odds of MDD at 9 and/or 18 Months in the Overall Sample ( $N=338$ )}

\begin{tabular}{|c|c|c|c|c|c|}
\hline Characteristic & $\begin{array}{c}\text { General Patient } \\
\text { Characteristics } \\
\text { OR }(95 \% \mathrm{CI})\end{array}$ & $\begin{array}{c}\text { Behavioral } \\
\text { Variables } \\
\text { OR (95\% CI) }\end{array}$ & $\begin{array}{c}\text { Biologic } \\
\text { Variables } \\
\text { OR }(95 \% \mathrm{CI})\end{array}$ & $\begin{array}{c}\text { Psychosocial } \\
\text { Variables } \\
\text { OR }(95 \% \mathrm{Cl})\end{array}$ & $\begin{array}{c}\text { Combined } \\
\text { Model } \\
\text { OR }(95 \% \mathrm{CI})\end{array}$ \\
\hline Age & $0.95^{\mathrm{a}}(0.92-0.99)$ & - & - & - & $0.96(0.92-1.01)$ \\
\hline Female & $1.64(0.77-3.46)$ & - & - & - & $1.29(0.50-3.30)$ \\
\hline White race & $1.16(0.55-2.45)$ & - & - & - & $0.96(0.37-2.46)$ \\
\hline Income & $0.99^{a}(0.98-1.01)$ & - & - & - & $1.00(0.98-1.01)$ \\
\hline Diet adherence & - & $0.87(0.70-1.08)$ & - & - & - \\
\hline Exercise adherence & - & $0.95(0.80-1.08)$ & - & - & - \\
\hline Hemoglobin $A_{1 c}$ & - & - & $1.22^{\mathrm{a}}(1.01-1.49)$ & - & $1.12(0.87-1.43)$ \\
\hline BMI & - & - & $1.04^{\mathrm{a}}(1.01-1.09)$ & - & $1.01(0.95-1.08)$ \\
\hline No. of comorbidities & - & - & $1.10(0.97-1.25)$ & - & - \\
\hline No. of diabetes complications & - & - & $0.87(0.65-1.18)$ & - & - \\
\hline Prior MDD & - & - & - & $4.15^{c}(1.97-8.73)$ & $4.19^{b}(1.79-9.79)$ \\
\hline No. of negative life events & - & - & - & $1.11^{\mathrm{a}}(1.01-1.24)$ & $1.10(0.98-1.24)$ \\
\hline Negative affect at baseline & - & - & - & $1.08^{c}(1.05-1.11)$ & $1.09^{c}(1.05-1.13)$ \\
\hline \multicolumn{6}{|c|}{$\begin{array}{l}\mathrm{Cl}=\text { confidence interval; } \mathrm{BMI}=\text { body mass index; hemoglobin } \mathrm{A}_{1 \mathrm{c}}=\text { glycated hemoglobin; } \mathrm{MDD}=\text { major depressive disorder; } \mathrm{OR}=\text { odds ratio. } \\
\text { a } \mathrm{P}<.05 . \\
\text { b } P=.001 .\end{array}$} \\
\hline
\end{tabular}


$(\mathrm{OR}=4.15 ; \mathrm{P}<.001)$, having more negative life events $(\mathrm{OR}=1.11 ; P$ $<.05)$, and having higher negative affect $(\mathrm{OR}=1.08 ; P<.001)$, all significantly and independently predicted MDD at 9 or 18 months.

\section{Combined Model}

We created a combined model that included the best predictors from each of the preceding analyses. This final model included hemoglobin $A_{1 c}, B M I$, negative life events, prior MDD, and current indicators of negative affect. In addition, all patient demographic characteristics were included as controls. Of these predictors, only current negative affect $(\mathrm{OR}=1.09 ; \mathrm{P}<.001)$ and prior MDD $(\mathrm{OR}=4.19 ; P=.01)$, each significantly and independently predicted the diagnosis of MDD at 9 or 18 months.

\section{Exploratory Analyses}

We conducted stratified analyses of participants who did not meet criteria for MDD at baseline but who displayed either a CES-D score of 10 or greater (moderate negative affect) $(n=121)$ or of 16 or greater (high negative affect) $(n=61)$ at baseline. Within the moderate negative affect subgroup, 7 patients developed MDD. As displayed in Table 3, a prior diagnosis of MDD $(\mathrm{OR}=3.10 ; P<.05)$, a high number of negative life events $(\mathrm{OR}=1.14 ; P<.05)$, a high level of hemoglobin $A_{1 c}(O R=1.36 ; P<.05)$, and a high $\mathrm{BMI}(\mathrm{OR}=1.09$; $P<.05)$, each significantly and independently predicted the subsequent development of MDD for this group. For the high negative affect subgroup (CES-D >16) 24 patients developed MDD. Only a high level of hemoglobin $\mathrm{A}_{1 \mathrm{c}}(\mathrm{OR}=1.63 ; P<.05)$ and a high number of negative life events $(\mathrm{OR}=1.21 ; P<.05)$ significantly and independently predicted the subsequent development of MDD for this group. One interaction term was significant, CES-D by prior MDD $(P<.05)$ : those with high current negative affect were more likely to have higher subsequent MDD scores than those with low current negative affect.

Results from an exploratory mediation analysis indicated that current negative affect partially mediated the relationship between prior MDD and subsequent MDD. Also, negative affect partially mediated the relationship between negative life events and subsequent MDD. ${ }^{15}$ As displayed in Figures 1 and 2, the odds ratios for the relationships between prior negative life events and subsequent MDD (at 9 and 18 months), as
Figure 1. Negative affect (CES-D) and prior MDD mediation model.

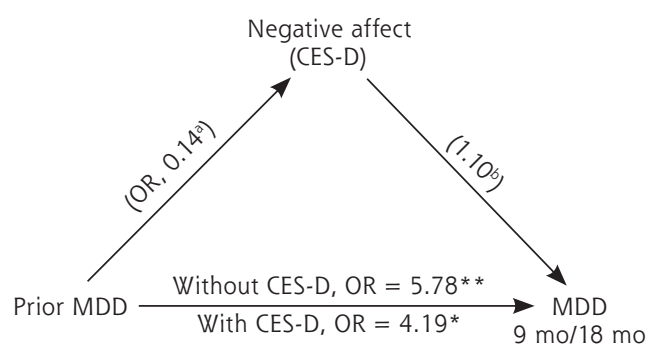

CES-D = Center for Epidemiological Studies-Depression Scale; MDD = major depressive disorder; NLE = Negative Life Events Scale; OR = odds ratio.

a $P<.01$.

b $P<.001$.

\section{Figure 2. Negative affect (CES-D) and negative} life events mediation model.

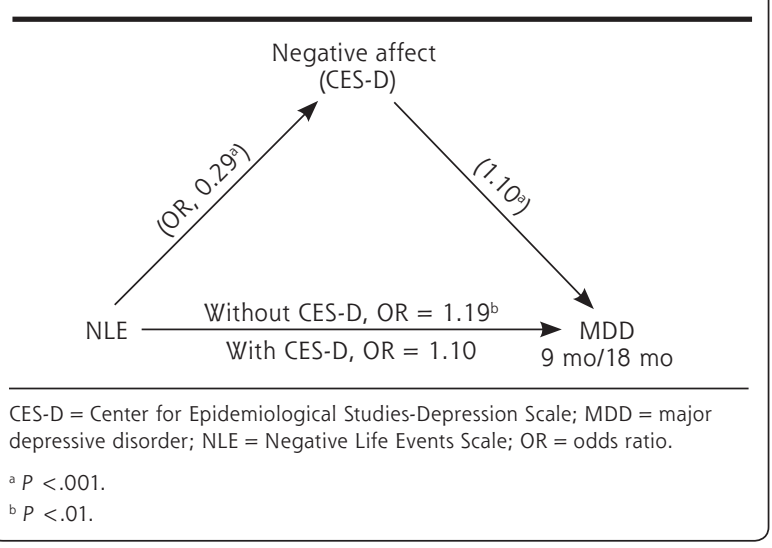


well as prior MDD and subsequent MDD (at 9 and 18 months), both decreased when negative affect was added to the model.

\section{DISCUSSION}

In this study, we sought to identify risk factors for developing MDD among demographic, behavioral, biologic, and psychosocial variables for patients with type 2 diabetes. We found that a prior diagnosis of MDD and current levels of negative affect at baseline significantly and independently predicted later MDD at 9 and/or 18 months. Our findings are similar to epidemiological reports of the association between negative affect and subsequent MDD in patients with diabetes. Katon and colleagues conducted a prospective study of patients with diabetes and found that the strongest predictor of developing MDD at the 5-year follow-up was baseline severity of depressive symptoms. ${ }^{2}$ In another long-term follow-up study, about $70 \%$ of patients with diabetes and major MDD or dysthymia had significant depressive symptoms previously for 2 years or more. ${ }^{17}$ The present study differs from these reports in that our baseline patient sample did not meet criteria for MDD; yet, even in this relatively lower risk sample, we found that negative affect remained a predictor of later MDD.

Although negative affect was the dominant predictor of MDD over time in the combined model, other important contributing factors were also involved. Results from the stratified analyses support this view. In examining the subgroup of patients with moderately increased negative affect, we found that in addition to having a prior diagnosis of MDD, the context of their daily life was also a salient predictor. Having more negative life events, elevated body weight, and more difficulty managing their hemoglobin $\mathrm{A}_{\mathrm{cc}}$ levels also significantly and independently predicted later development of MDD. In the subgroup of patients with highly elevated negative affect, again contextual negative life events and management of their hemoglobin $\mathrm{A}_{\mathrm{1c}}$ levels independently predicted later development of MDD. These findings suggest that it may be helpful clinically to view negative affect within the context of broader life and chronic disease factors, rather than focus exclusively on affective status alone, divorced from real-world contributing conditions.

In the exploratory mediation analyses, we found that negative affect partially mediated the relationships between negative life events and future MDD, as well as prior MDD and future MDD. In essence, having high negative life events and prior MDD influenced both negative affect and subsequent onset of MDD.

Thus, we suspect a process by which a variety of problematic life stresses, diabetes-related variables, and history combine to yield the kinds of negative emotional symptoms captured by the CES-D. In combination, these variables predict MDD over time, with negative affect reflecting the poor mood generated by these other contextual life events and circumstances, eg, negative life events, poor glycemic control. This interpretation allows a focus on a variety of life context factors and not on negative affect alone, thus expanding our understanding of the processes involved and expanding the range of potential interventions that might be relevant in a primary care setting.

There is a large literature documenting the association between stressful life events and risk of depression in the general population. ${ }^{18}$ This relationship has been documented for both acute ${ }^{19}$ and chronic stresses. ${ }^{20}$ Less work has been done, however, showing the ways in which additonal chronic and acute disease stressors are related to disease management and negative affect for patients with chronic disease.

Our findings have important implications for clinical care. Although repeated assessment of negative affect over time among patients with type 2 diabetes is warranted, it may also be helpful to expand the lens of inquiry to include a discussion of disease-related and other life stressors that may be contributing to poor mood when it is detected at screening. Doing so directs attention not only to affective status but also to the conditions associated with the observed dysphoria, eg, diabetes distress. Awareness of these contributing factors helps initiate a conversation about life pressures, coping skills, and needed resources that can help prevent the development of subsequent MDD, rather than focusing on psychiatric diagnosis or mood alone.

There are several limitations to these findings. First, we systematically explored a relatively large number of predictors with a sample of modest size ; therefore, we may not have had sufficient power to detect associations between some variables and developing MDD. Second, the 18-month period that we observed patients may not have been long enough to capture a sufficiently large group of patients that developed MDD. Additionally, had the follow-up been longer, more cases of MDD may have been detected that captured a different configuration of predictive variables. Even so, we obtained a prevalence for patients who developed MDD that corresponds to the literature (roughly 12\%). Third, our effect sizes were modest, although we were still able to demonstrate statistically significant linkages, which we feel add to the understanding of depression in patients with type 2 diabetes. Fourth, because of a small sample size, we had little power to detect differential predictors of MDD at 9 and 18 months after baseline in subgroups of patients with moderate CES-D scores (10 to 15$)$ and high CES-D scores (16 and greater). Instead, 
we reported significant results between overlapping groups with CES-D scores of $>9$ and $>15$. Analyses not reported, however, showed similar trends with the smaller subsamples.

Despite these limitations, we identified several significant, independent predictors of MDD that can assist in identifying patients with diabetes who may be at risk. The most important clinical implication is the recognition that negative affect does not occur in isolation from other aspects of a patient's social and disease-related contexts. When patients have even moderate levels of increased depressive symptoms, it may be helpful to inquire about other life stressors and chronic disease management. Devoting attention to the contextual stressors that occur in patients' lives and providing appropriate direct interventions or referral to services that might help reduce the negative impact of these stressors may have a positive effect on both their emotional status and their disease management.

To read or post commentaries in response to this article, see it online at http://www.annfammed.org/cgi/content/full/9/2/115.

Key words: Type 2 diabetes mellitus; stress psychological; depressive disorder

Submitted April 22, 2010; submitted, revised, September 22, 2010; accepted October 5, 2010.

Funding support: This research was supported by grants DK062732 and DK061937 from the National Institute of Diabetes, Digestive and Kidney Disease.

Acknowledgments: The following San Francisco and surrounding bay area medical groups and diabetes education centers collaborated in this research: Alta Bates Diabetes Education Center, Brown and Toland Medical Group, California Pacific Diabetes Education Center, Hill Physicians Medical Group, Marin IPA, St Luke's Diabetes Education Center, St Mary's Medical Center, University of California, San Francisco Hospital and Clinics.

\section{References}

1. Katon WJ, Rutter C, Simon G, et al. The association of comorbid depression with mortality in patients with type 2 diabetes. Diabetes Care. 2005;28(11):2668-2672.

2. Katon W, Russo J, Lin EH, et al. Depression and diabetes: factors associated with major depression at five-year follow-up. Psychosomatics. 2009;50(6):570-579.
3. Golden SH, Lazo M, Carnethon M, et al. Examining a bidirectional association between depressive symptoms and diabetes. JAMA. 2008;299(23):2751-2759.

4. Janes GR. Ambulatory Medical Care for Diabetes. 2nd ed. Bethesda, MD: National Institutes of Health; 1995.

5. Skovlund SE. Patient-reported assessments in diabetes care: clinical and research applications. Curr Diab Rep. 2005;5(2):115-123.

6. Ruben RR, Ciechanowski P, Egede LE, Lin E, Lustman PJ. Recognizing and treating depression in patients with diabetes. Curr Diab Rep. 2004;4(2):119-125.

7. Pouwer F. Should we screen for emotional distress in type 2 diabetes mellitus? Nat Rev Endocrinol. 2009;5(12):665-671.

8. Fisher L, Mullan JT, Skaff MM, Glasgow RE, Areán P, Hessler D. Predicting diabetes distress in patients with Type 2 diabetes: a longitudinal study. Diabet Med. 2009;26(6):622-627.

9. Fisher L, Skaff MM, Mullan JT, Areán P, Glasgow R, Masharani U. A longitudinal study of affective and anxiety disorders, depressive affect and diabetes distress in adults with Type 2 diabetes. Diabet Med. 2008;25(9):1096-1101.

10. Toobert DJ, Hampson SE, Glasgow RE. The summary of diabetes self-care activities measure: results from 7 studies and a revised scale. Diabetes Care. 2000;23(7):943-950.

11. Radloff LS. The CES-D scale: A self-report depression scale for research in the general population. Appl Psychol Meas. 1977;1(3): 385-401.

12. Turner RJ, Wheaton B, Lloyd DA. The epidemiology of social stress. Am Sociol Rev. 1995;60(1):104-125.

13. Wittchen HU. Reliability and validity studies of the WHOComposite International Diagnostic Interview (CIDI): a critical review. J Psychiatr Res. 1994;28(1):57-84.

14. Hosmer DW, Lemeshow S. Applied Logistic Regression. New York, NY: Wiley; 2000.

15. Baron RM, Kenny DA. The moderator-mediator variable distinction in social psychological research: conceptual, strategic, and statistical considerations. J Pers Soc Psychol. 1986;51(6):1173-1182.

16. Fisher L, Mullan JT, Areán P, Glasgow RE, Hessler D, Masharani U. Diabetes distress but not clinical depression or depressive symptoms is associated with glycemic control in both cross-sectional and longitudinal analyses. Diabetes Care. 2010;33(1):23-28.

17. Lustman PJ, Griffith LS, Freedland KE, Clouse RE. The course of major depression in diabetes. Gen Hosp Psychiatry. 1997;19(2): 138-143.

18. Hammen C. Stress and depression. Annu Rev Clin Psychol. 2005; 1:293-319.

19. Kendler KS, Karkowski LM, Prescott CA. Stressful life events and major depression: risk period, long-term contextual threat, and diagnostic specificity. J Nerv Ment Dis. 1998;186(11):661-669.

20. Hammen C, Kim EY, Eberhart NK, Brennan PA. Chronic and acute stress and the prediction of major depression in women. Depress Anxiety. 2009;26(8):718-723. 